\title{
Constraining the sensitivity of iodide adduct chemical ionization mass spectrometry to multifunctional organic molecules using the collision limit and thermodynamic stability of iodide ion adducts
}

\author{
Felipe D. Lopez-Hilfiker ${ }^{1}$, Siddarth Iyer ${ }^{2}$, Claudia Mohr ${ }^{1}$, Ben H. Lee ${ }^{1}$, Emma L. D'Ambro' ${ }^{1,3}$, Theo Kurtén ${ }^{2}$, and \\ Joel A. Thornton ${ }^{1}$ \\ ${ }^{1}$ Department of Atmospheric Sciences, University of Washington, Seattle, 98195 WA, USA \\ ${ }^{2}$ Department of Chemistry, University of Helsinki, Helsinki, Finland \\ ${ }^{3}$ Department of Chemistry, University of Washington, Seattle, 98195 WA, USA
}

Correspondence to: Joel A. Thornton (thornton@atmos.washington.edu)

Received: 15 September 2015 - Published in Atmos. Meas. Tech. Discuss.: 23 October 2015

Revised: 11 February 2016 - Accepted: 18 February 2016 - Published: 6 April 2016

\begin{abstract}
The sensitivity of a chemical ionization mass spectrometer (ions formed per number density of analytes) is fundamentally limited by the collision frequency between reagent ions and analytes, known as the collision limit, the ion-molecule reaction time, and the transmission efficiency of product ions to the detector. We use the response of a timeof-flight chemical ionization mass spectrometer (ToF-CIMS) to $\mathrm{N}_{2} \mathrm{O}_{5}$, known to react with iodide at the collision limit, to constrain the combined effects of ion-molecule reaction time, which is strongly influenced by mixing and ion losses in the ion-molecule reaction drift tube. A mass spectrometric voltage scanning procedure elucidates the relative binding energies of the ion adducts, which influence the transmission efficiency of molecular ions through the electric fields within the vacuum chamber. Together, this information provides a critical constraint on the sensitivity of a ToF-CIMS towards a wide suite of routinely detected multifunctional organic molecules for which no calibration standards exist. We describe the scanning procedure and collision limit determination, and we show results from the application of these constraints to the measurement of organic aerosol composition at two different field locations.
\end{abstract}

\section{Introduction}

The photochemical oxidation of volatile organic compounds (VOCs) in the atmosphere generates a wide array of multifunctional organic molecules which contribute to the formation of secondary organic aerosol (SOA), hydroxyl radical sources and sinks, and the cycling and fate of reactive nitrogen. Determination of the identities of these organics, and their abundance in the atmosphere, has remained an analytical challenge because of the inherent complexity of the chemical system, which involves a multitude of precursors and significantly more oxidation products (Bertram et al., 2009; Goldstein and Galbally, 2007). Chemical ionization mass spectrometry (CIMS) has become increasingly utilized for the measurement of these types of compounds (Bertram et al., 2011; Brophy and Farmer, 2015; Fortner et al., 2004; Hearn and Smith, 2004; Holzinger et al., 2010; Huey et al., 1995; Jokinen et al., 2012; Jordan et al., 2009; Lee et al., 2014; Lopez-Hilfiker et al., 2014; Slusher, 2004; Veres et al., 2008, 2010; Yatavelli et al., 2012; You et al., 2014; Yu and Lee, 2012). Typically, a specific reagent ion is generated using a radioactive ion source, $\mathrm{X}$-rays, or corona discharge, and then mixed with ambient air for a fixed time. Ion-molecule reactions then lead to the formation of product ions which are separated and counted with a mass spectrometer. Common ion-molecule reaction mechanisms include ligand switching (adduct formation), reactive electron transfer, or proton transfer/abstraction. The benefits of CIMS include linearity, reproducibility, sensitivity with some degree of se- 
lectivity, and high time resolution without sample preparation or handling. General disadvantages of CIMS include a lack of isomer or isobaric separation and thus structural information without the coupling of addition separation dimensions, and the range of potential sensitivities which require calibration with authentic standards.

Recently, chemical ionization has been coupled to field deployable time-of-flight mass spectrometers (ToF-MS) such as the Tofwerk AG high-resolution version, commonly referred to as the HRToF-CIMS (Aljawhary et al., 2013; Huey et al., 1995; Jokinen et al., 2012; Junninen et al., 2010; Lee et al., 2014; Lopez-Hilfiker et al., 2014; Yatavelli et al., 2012). As a result, hundreds of oxidized organic compounds are now routinely detected in ambient air or photo-oxidation experiments in the laboratory with a single instrument. A major limitation of these instruments thus far is that calibration of the instrument response to many of the detected ions is impossible, as either the sheer number of calibrations required is unrealistic, or calibration standards do not exist.

Herein, we present the maximum sensitivity of an HRToFCIMS using the collision limit for iodide adduct chemical ionization which is becoming widely used by the atmospheric chemistry community (Aljawhary et al., 2013; Huey et al., 1995; Kercher et al., 2009; Lee et al., 2014). We also present an ion adduct declustering scanning procedure which experimentally determines the relative binding energies of the detected ion adducts and therefore their approximate sensitivity. The combination of declustering scanning to determine effective binding enthalpies, which can be compared with theoretical estimates from quantum mechanical calculations, along with the experimentally determined collision limit provides an approximate calibration for many compounds in the mass spectrum which would otherwise be impossible to obtain by traditional methods.

\section{Iodide ToF-CIMS sensitivity to organics}

Iodide adduct chemical ionization mass spectrometry has been described in detail previously (Huey et al., 1995; Kercher et al., 2009; Lee et al., 2014). As summarized in Eq. (1), for adduct ionization, there are essentially two components to the instrument sensitivity that will be specific to a molecule: (i) the rate at which product ions are formed via reagent ion-molecule reactions over the fixed interaction time, and (ii) the transmission of the molecular ion to the detector. In Eq. (1), $S_{i}$ is the sensitivity observed for reaction time $t, k_{\mathrm{f}}$ is the product ion formation rate constant, $\left[I^{-}\right]$ is the concentration of the reagent ions in the ion molecule region (IMR), and $T^{i}$ is the ion-specific transmission efficiency, which depends upon the ion mass-to-charge $(\mathrm{m} / Q)$, net electric field strength of the transfer optics $(\varepsilon)$, and the adduct ion binding energy $\left(B^{i}\right)$.

$$
\begin{aligned}
S_{i} & =\int_{0}^{t} k_{\mathrm{f}}\left[I^{-}\right] \mathrm{d} t \times T^{i}\left(\frac{m}{Q}, B^{i}\right) \\
& =\text { product ion formation } \times \text { transmission }
\end{aligned}
$$

A neutral molecule that forms a strongly bound cluster with iodide at the collision limit should be detected with relatively high sensitivity given that it will survive transmission through the ion optics which inherently impart energy to the ions via electric fields. In contrast, a molecule might form an iodide adduct at the collision limit, but be so weakly bound that it is not detected due to collision-induced dissociation ("declustering") during transit through the vacuum chamber. Thus, knowledge of a cluster's binding energy and the collision-limited formation rate can provide a means to further constrain the instrument's sensitivity to a broader range of compounds it detects, even if standards do not exist. Experimental constraints on binding energies and collisionlimited product ion formation rates are discussed below.

\subsection{Collision limit determination of the UW-ToF-CIMS}

We have calibrated the iodide adduct ToF-CIMS to many organic and inorganic molecules including hydroxyhydroperoxides, multifunctional acids, diols, triols, tetrols, nitrated aromatics and other oxidized organic molecules in an effort to constrain the instrument response to a variety of different functionalities (Lee et al., 2014). As expected, given constraints imposed by ion-molecule collision frequencies, we empirically find that there is a "maximum sensitivity", which for iodide-organic clusters in our instrument is $\sim 19$ $22 \mathrm{cps} \mathrm{pptv}^{-1}$ (per million cps of reagent ion) (see, e.g., Lee et al., 2014). As discussed below, this limit is also consistent with the experimental ion-molecule collision limit of our instrument.

Huey et al. (1995) first showed that dinitrogen pentoxide $\left(\mathrm{N}_{2} \mathrm{O}_{5}\right)$ reacts with iodide ions at the collision limit (Huey et al., 1995). We therefore use this reaction to determine the upper-limit sensitivity of our instrument (to $\mathrm{N}_{2} \mathrm{O}_{5}$ ) given that the number of product ions detected cannot exceed those produced by the number of iodide- $\mathrm{N}_{2} \mathrm{O}_{5}$ collisions occurring within the interaction time of ions and molecules.

We generate isotopically labeled ${ }^{15} \mathrm{~N}_{2} \mathrm{O}_{5}$ by reacting excess ${ }^{15} \mathrm{NO}_{2}$ (Scott-Marin) with ozone leading to the formation of ${ }^{15} \mathrm{NO}_{3}$ and ${ }^{15} \mathrm{~N}_{2} \mathrm{O}_{5}$ during transit down a Teflon reaction cell held at $207 \mathrm{kPa}$ above ambient pressure by a glass capillary. The output can be modeled (Bertram et al., 2009) and has been independently verified by other techniques such as thermal-dissociation laser-induced fluorescence or cavity ring-down spectroscopy (Brown et al., 2001; Day et al., 2002). We use the independently calibrated output concentrations as inputs into the mass spectrometer and monitor the response.

$\mathrm{N}_{2} \mathrm{O}_{5}$ reacts with iodide ions at the collision limit (Huey et al., 1995) but via two channels (Kercher et al., 2009). One 


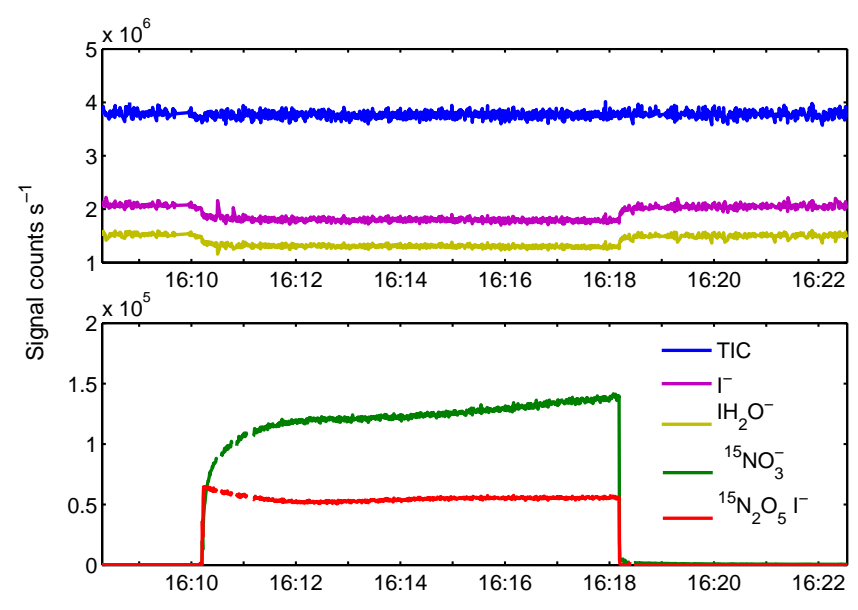

Figure 1. An example of collision-limited sensitivity determination of the ToF-CIMS. Top: the reagent ions and sum of total ions during the addition of high concentrations of ${ }^{15} \mathrm{~N}_{2} \mathrm{O}_{5}$ to the inlet of the ToF-CIMS. By calibrating the output of our $\mathrm{N}_{2} \mathrm{O}_{5}$ source independently (in this case by the UC, Berkeley TD-LiF instrument, Day et al., 2002), we are able to derive the collision-limited sensitivity of the instrument by adding the two detection channels as described in the text (Day et al., 2002; Huey et al., 1995). As total ion current (TIC) remains constant during the experiment despite the depletion of reagent ions $\left(\mathrm{I}^{-}+\mathrm{IH}_{2} \mathrm{O}-\right)$ the mass transmission efficiency between 63 and $237 \mathrm{Th}$ is therefore constant.

channel is the formation of an ion-molecule adduct between $\mathrm{N}_{2} \mathrm{O}_{5}$ and iodide $\mathrm{I}\left(\mathrm{N}_{2} \mathrm{O}_{5}\right)^{-}$. This cluster may simply be a stable intermediate on the way to the lowest energy reaction products $\mathrm{NO}_{3}^{-}$and $\mathrm{INO}_{2}$, but it is detected as a major product under weak electric field settings (weak declustering) in the ion optics used to transmit ions through the vacuum chamber to the mass separation region (Kercher, et al., 2009). The other channel results in $\mathrm{NO}_{3}^{-}+\mathrm{INO}_{2}$, presumably from the dissociation of the iodide adduct. Its contribution can be enhanced by increasing the strength of the electric fields in the atmospheric pressure interface (APi) of the mass spectrometer (Kercher et al., 2009). In the work of Huey et al. (1995) only $\mathrm{NO}_{3}^{-}$is observed due presumably to a combination of low pressure in the ion-molecule reaction drift tube, where the iodide $-\mathrm{N}_{2} \mathrm{O}_{5}$ collision complex might not be stabilized, and there are strong electric fields in the vacuum chamber. Therefore, to track the formation of product ions from the reaction of $\mathrm{I}^{-}$with $\mathrm{N}_{2} \mathrm{O}_{5}$, we add the product ion signals from the two detection channels $\left(\mathrm{NO}_{3}^{-}\right.$and $\left.\mathrm{IN}_{2} \mathrm{O}_{5}^{-}\right)$. An example time series of this type of experiment is shown in Fig. 1.

As the $\mathrm{N}_{2} \mathrm{O}_{5}$ product ions are detected at different massto-charge ratios ( 63 vs. $237 \mathrm{Th}$ ), the absolute count rate of the sum of the two ion signals could be influenced by mass-dependent ion transmission through the ion optics of the instrument. We therefore measured the mass-dependent transmission of our instrument by adding large quantities of known compounds with varying molecular mass to the ionization region (Huey et al., 1995). This method assumes that the total number of charges (ions) in the ionization region remains unchanged over short time periods (controlled by the activity of the ${ }^{210} \mathrm{Po}$ ); therefore, any changes to the total number of ions measured at the detector is due to the varying efficiency with which ions having different masses are transmitted through the mass spectrometer. By measuring the relative change in total ions detected as a function of mass to charge $(m / Q)$, a linear system of equations can be solved to derive the transmission efficiency as a function of mass to charge. The transmission efficiency depends on ion optic settings, primarily the two quadrupole ion guides which act as band-pass filters. The lower mass cutoff is most important for our sensitivity determination as ${ }^{15} \mathrm{NO}_{3}(63 \mathrm{Th})$ is near the low end of the mass transmission window. We tune the transmission function to be as flat as possible by adjusting the radio frequency, amplitude and axial voltage gradient along the quadrupole ion guides. As a result, in the mass range of interest (63-237 Th), the transmission efficiency is approximately constant in our instrument as evidenced by the ion closure shown in the top panel of Fig. 1 (top panel) during $\mathrm{N}_{2} \mathrm{O}_{5}$ additions.

Dividing the transmission-efficiency-corrected sum of $\mathrm{NO}_{3}^{-}$and $\mathrm{IN}_{2} \mathrm{O}_{5}^{-}$count rates by the $\mathrm{N}_{2} \mathrm{O}_{5}$ concentration (pptv) sampled, we calculate the total sensitivity to $\mathrm{N}_{2} \mathrm{O}_{5}$ to be 22-26 cps ppt ${ }^{-1}$ per million reagent ions. Given that $\mathrm{I}^{-}$and $\mathrm{N}_{2} \mathrm{O}_{5}$ react at the collision limit, and assuming there are no other product ions (we detect no others with the ToFCIMS), then this sensitivity represents the maximum possible sensitivity for compounds with collision cross sections similar to $\mathrm{N}_{2} \mathrm{O}_{5}$. As noted above, this estimate is also consistent with an empirically determined upper-limit sensitivity for organic compounds, in that we have yet to measure a sensitivity above this value. Some of the organic compounds to which we have calibrated that are near this collisionlimited sensitivity include isoprene-derived 2-methyl tetrols $\left(19 \mathrm{cps} \mathrm{ppt}^{-1}\right)$, dipentaerythritol $\left(22 \mathrm{cps} \mathrm{ppt}^{-1}\right)$, malonic acid $\left(19 \mathrm{cps} \mathrm{ppt}^{-1}\right)$ and levoglucosan $\left(20 \mathrm{cps} \mathrm{ppt}^{-1}\right)$.

\subsection{Distribution iodide adduct binding energies}

In our instrument, organic molecules are nearly exclusively detected as molecular clusters with iodide. However, outside of a few of the simplest carboxylic acids, very few binding energies of organic compounds with iodide have been measured or calculated. Binding energies calculated using quantum chemical methods provide valuable information, but carrying out the computationally expensive calculations for the 100 s of molecular ions typically identified in our spectra is not feasible, especially when molecular structure is unknown. Therefore, to constrain the effective binding energies of the actual multifunctional organics that are measured, we scan the electric field strength within the transfer optics in real time while measuring a steady-state distribution of organic compounds. These scans experimentally determine the electric field strength required to break apart the iodide- 


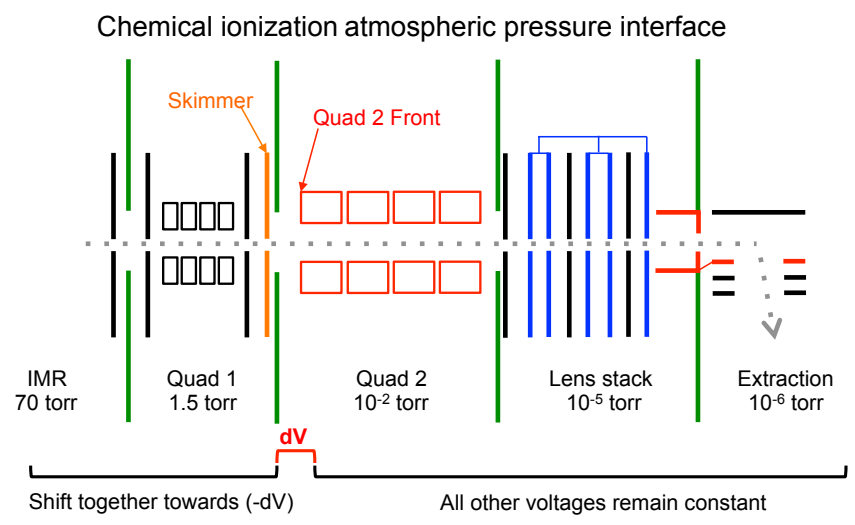

Figure 2. A schematic of the voltages in the APi region of the Tofwerk ToF-MS. The region of the mass spectrometer that we conduct declustering scanning is between the skimmer (orange) and the entrance to the second quadrupole (red). All voltages upstream of the skimmer are moved incrementally towards more negative voltages to create a stronger declustering field while keeping mass transmission effects constant by keeping the voltage gradients across each quadrupole constant, typically in steps of $-1 \mathrm{~V}$. Iodide adducts which are formed in the ion molecule region (IMR) interact with the changing electric fields and dissociate based on their ion-molecule binding energy.

organic adducts, which, in turn, are directly related to the binding energy of the adduct.

To assess this approach, we used a steady-state atmospheric simulation chamber at the University of Washington to generate a wide range of oxidized organics from the reaction of $\alpha$-pinene in the presence of ozone and $\mathrm{NO}_{x}$ (Lopez-Hilfiker et al., 2015). While sampling this mixture, we scanned the voltage difference $(\mathrm{d} V)$ between the skimmer and the entrance to the second quadrupole ion guide of the mass spectrometer (see Fig. 2 for schematic). We call these declustering scans because - by increasing $\mathrm{d} V$ - we systematically increase the collisional energy of the iodide-organic adducts above our normal operating $\mathrm{d} V$ until the adducts dissociate, mostly into $\mathrm{I}^{-}$and a neutral organic molecule. An example of this type of experiment is shown in Fig. 3a, where normalized ion count rates are plotted as a function of $\mathrm{d} V$. During a declustering scan, all potentials upstream of the second quadrupole are moved together towards more negative voltages such that the electric field and therefore the declustering strength is incrementally changed while maintaining a constant gradient across the quadrupoles to avoid simultaneous changes in the mass transmission function, which depends upon the axial voltage gradient along the quadrupole rods (see Fig. 2 schematic).

We show the survival of a representative set of iodide adducts as a function of electric field strength in Fig. 3a. A variety of behaviors are observed; however, all adducts follow a similar sigmoidal response to $\mathrm{d} V$, as expected for a threshold-driven process. We find that some adducts (e.g.,

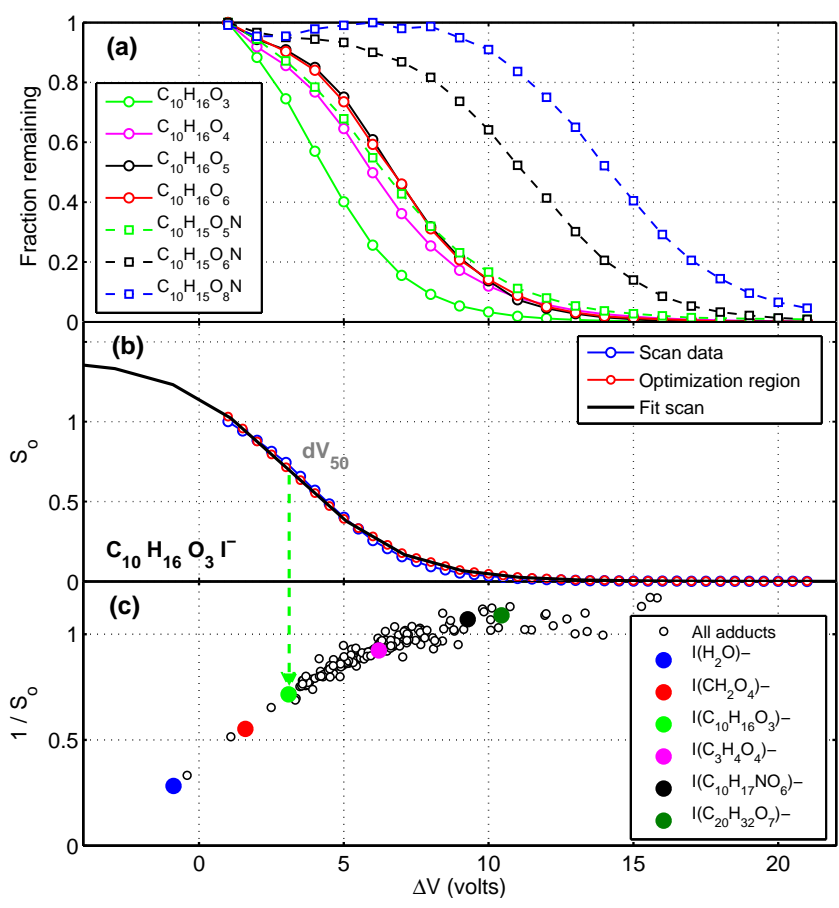

Figure 3. Effective binding energy. Top: declustering scans of gasphase products measured from the reaction of $\alpha$-pinene in the presence of ozone and $\mathrm{NO}_{x}$. Some iodide adducts dissociate rapidly during the first few voltage steps. Multifunctional nitrates and highly oxidized $\mathrm{C}_{10}$ molecules show no dependence on the initial voltage steps before dissociating. We infer these compounds to be strongly bound to $\mathrm{I}^{-}$, and therefore likely detected at a high sensitivity. Middle: an example of a non-linear least-squares fit to the declustering scan is shown for $\mathrm{C}_{10} \mathrm{H}_{16} \mathrm{O}_{3} \mathrm{I}^{-}$. The raw scan data are shown in blue circles, the region of optimization for the fit is shown in red, and the extrapolated scan curve is shown in black, constraining $S_{o}$. Bottom: the results of fitting declustering scans $\left(1 / S_{O}\right)$ shows a plateauing effect as a function of $\mathrm{d} V_{50}$. We use $\mathrm{d} V_{50}$ as a measure of the relative binding energy between compounds. In colored dots, specific molecules are shown which span the binding enthalpy space.

simple monocarboxylic acids, diols) are rapidly dissociated during the first few voltage steps, while larger multifunctional organics (inferred from the $\mathrm{O} / \mathrm{C}$ ratio) tend to survive to higher potentials during the scan (e.g., multifunctional organic nitrates in Fig. 3a). The observation that the larger, multifunctional molecules (e.g., $\mathrm{C}_{10} \mathrm{H}_{15} \mathrm{O}_{8} \mathrm{~N}$ ) are detected with the same efficiency during the first few voltage steps implies that they are bound to iodide with sufficient binding energy to be efficiently transmitted by the normal operation of the ion optics in the mass spectrometer and are therefore likely to be detected at a sensitivity that depends only on their formation rate, which for lack of a better constraint we would assume to be at the collision limit.

Even at our weakest electric field settings, many iodideorganic adducts are partially declustered, and thus the true sigmoidal declustering scan curve is not observed. In these 
cases, we calculate an effective maximum sensitivity by using a custom non-linear least-squares fitting algorithm to determine the extent to which the ion adduct has been declustered during transit through the mass spectrometer's atmospheric pressure interface (APi). The fitting algorithm uses a characteristic sigmoidal shape with variable amplitude, $S_{o}$, and location of the voltage at half signal maximum $\left(\mathrm{d} V_{50}\right)$. If there are isomers or isobaric compounds that contribute significantly to the ion signal but have different iodide binding energies, then the declustering scan should not have a sigmoidal shape. While this information could be useful with a highly resolved $\mathrm{d} V$ scan, and is reflected in the fit error, herein we remove ion adducts with mean square residual $>10 \%$ from the analysis. $S_{o}$ is the relative signal that would be detected under weaker electric field strengths than we can operate the instrument $(\mathrm{d} V<1 \mathrm{~V}) . S_{o}$ is not a measure of the actual sensitivity, which is generally unknown, but instead is a measure of the extent to which declustering during transmission to the detector affects the actual sensitivity. The $\mathrm{d} V_{50}$ is a measure of relative binding enthalpy. Iodide adducts that are tightly bound survive to higher voltage gradients and therefore have a higher $\mathrm{d} V_{50}$ than more weakly bound adducts.

Pinonic acid (assumed to be measured as the $\mathrm{C}_{10} \mathrm{H}_{16} \mathrm{O}_{3} \mathrm{I}^{-}$ ion) is an example of a compound for which a true sigmoidal curve is not observed (see Fig. 3b). The fit for pinonic acid implies that the sensitivity would be enhanced if weaker declustering conditions existed (e.g., $1.4=S_{o}>1$ ). The sensitivity of the instrument to pinonic acid, calibrated by an authentic standard is $15 \mathrm{cps} \mathrm{ppt}^{-1}$. Therefore, if weaker declustering conditions existed in the instrument, transmissionoptimized sensitivity for pinonic acid would be $15 \times 1.4=$ $21 \mathrm{cps} \mathrm{ppt}^{-1}$. This value is near the collision limit determined by calibration to $\mathrm{N}_{2} \mathrm{O}_{5}$, which suggests that the iodidepinonic adduct is formed at near the collision limit in the IMR, but it is partially declustered during transit through the ion optics of the mass spectrometer, resulting in a lower observed sensitivity during normal operation settings (e.g., $S_{\text {obs }}<S_{o}$ ).

In Fig. 3c we show similar voltage scanning fit results for all iodide-organic adducts (black circles) identified in the mixture produced from $\alpha$-pinene ozonolysis in the presence of $\mathrm{NO}_{x}$. We find that $1 / S_{o}$, which is related to the maximum possible transmission for each compound, plateaus with increasing $\mathrm{d} V_{50}$, suggesting that iodide adducts with a $\mathrm{d} V_{50}$ $\sim 6 \mathrm{~V}$ or higher, are sufficiently bound to transit the ion optics without significant declustering losses. Adducts having $\mathrm{d} V_{50}>6 \mathrm{~V}$ are composed of highly functionalized organics (e.g., Fig. 3c: $\mathrm{C}_{10} \mathrm{H}_{17} \mathrm{NO}_{6}$ (black) and $\mathrm{C}_{20} \mathrm{H}_{32} \mathrm{O}_{7}$ (green)), which is consistent with the more strongly bound iodide adducts generally involving one or more hydrogen bonds from a polar hydroxy, hydroperoxy, or carboxylic acid group. We also expect that these compounds are likely formed at near the collision limit as steric effects are unlikely to signif-
Table 1. Compounds used to determine the relationship between $\mathrm{d} V_{50 \%}$ and binding enthalpy derived from quantum calculations (Iyer et al., 2016) at the DLPNO-CCSD(T)//PBE-aug-cc-pVTZ-PP level. The relationship is approximately linear $R^{2}=0.9$ (see Fig. 4). For details see text.

\begin{tabular}{llrr}
\hline Compound & Composition & $\begin{array}{r}\text { Binding enthalpy } \\
\left(\mathrm{kcal} \mathrm{mol}^{-1}\right)\end{array}$ & Fit d $V_{50}(\mathrm{~V})$ \\
\hline Glycolic acid & $\mathrm{C}_{2} \mathrm{H}_{4} \mathrm{O}_{3}$ & -21.1 & 4.70 \\
Glyoxylic acid & $\mathrm{C}_{2} \mathrm{H}_{2} \mathrm{O}_{3}$ & -20.8 & 4.29 \\
Malonic acid & $\mathrm{C}_{3} \mathrm{H}_{4} \mathrm{O}_{4}$ & -27.8 & 6.21 \\
Formic acid & $\mathrm{CH}_{2} \mathrm{O}_{2}$ & -23.9 & 5.80 \\
Acetic acid & $\mathrm{C}_{2} \mathrm{H}_{4} \mathrm{O}_{2}$ & -17.4 & 4.10 \\
Succinic acid & $\mathrm{C}_{4} \mathrm{H}_{8} \mathrm{O}_{4}$ & -27.6 & 6.19 \\
Nitric acid & $\mathrm{HNO}_{3}$ & -22.2 & 5.50 \\
Nitrous acid & $\mathrm{HONO}$ & -18.7 & 4.56 \\
\hline
\end{tabular}

icantly limit their formation rate, but this hypothesis remains to be tested.

\subsection{Relationship of $\mathrm{d} V_{50}$ to quantum-chemical-derived binding energies}

Figure 4 shows the relationship between iodide adduct binding enthalpies from quantum chemical calculations, the $\mathrm{d} V_{50}$ values determined from the fits to the declustering scans (see also Table 1). Assuming the linear relationship $\left(R^{2}=0.92\right)$ between the subset of compounds for which we have quantum chemical calculations and experimental determinations holds, the derivation of the binding energy from declustering scans for hundreds of compounds simultaneously is then possible without explicit knowledge of the functional groups or molecular geometry, which is required for quantum calculations. We have shown in a related article that there is a reasonable relationship between theoretical binding enthalpies and measured sensitivity (Iyer et al., 2016). Therefore, by constraining the relationship between quantum calculations and measured scan shape for a subset of compounds, we can use the measured $\mathrm{d} V_{50}$ to estimate the binding enthalpy, and thus instrument sensitivity, for compounds that are too computationally intensive or for which we lack knowledge of molecular structure or cartesian geometries necessary for optimization. As noted above, the binding enthalpy of an adduct alone does not necessarily determine overall sensitivity. The rate of adduct formation and transmission through the mass spectrometer are both important components of the overall sensitivity.

\section{Application to atmospheric organic aerosol}

As an example of the potential application of the above constraints, we apply the collision-limit sensitivity of $22 \mathrm{cps} \mathrm{ppt}^{-1}$ per million reagent ions to organic compounds detected generally as $\mathrm{C}_{x} \mathrm{H}_{y} \mathrm{O}_{z} \mathrm{~N}_{0-1} \mathrm{I}^{-}$upon temperatureprogrammed thermal desorption of ambient submicron aerosol using a FIGAERO-HRToF-CIMS (Lopez-Hilfiker et 


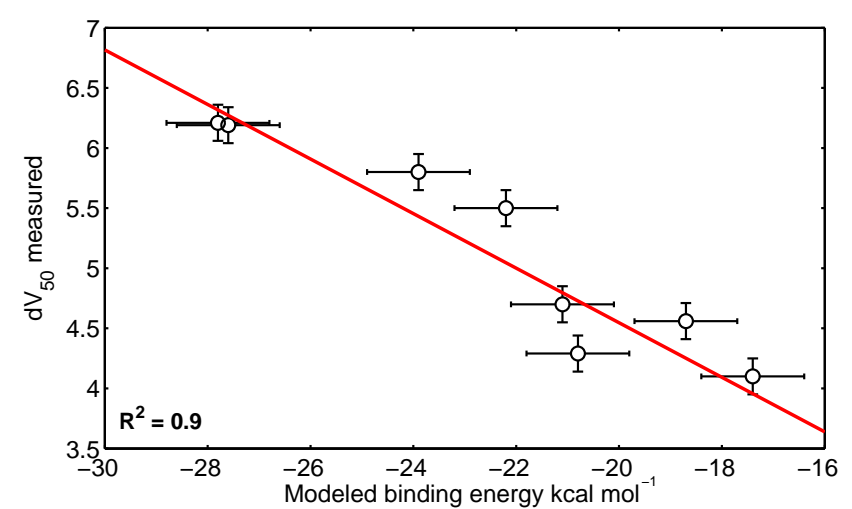

Figure 4. The relationship between calculated binding enthalpy and $\mathrm{d} V_{50}$ is shown for compounds which were observed from the oxidation of $\alpha$-pinene in the presence of $\mathrm{NO}_{x}$ and for which quantum calculations have been performed (see Table 1). The modeled and measured relationship allows estimation of the binding energy of molecules which are too complex or computationally intensive to calculate. Also shown in red is a linear least-squares fit to the data $\left(R^{2}=0.9\right)$.

al., 2014). We compare the resulting sum total mass loadings of all molecular components with the submicron organic aerosol mass concentrations measured by an aerosol mass spectrometer (AMS) (DeCarlo et al., 2006). Applying the collision-limited sensitivity to all organic-iodide adducts to which we have not explicitly calibrated (vast majority of ions) results in a lower limit to mass concentrations measured by the FIGAERO HR-ToF-CIMS. Figure 5 shows the result of this comparison for two different locations: (1) a polluted region in the southeast United States (Brent, Alabama), which is dominated by isoprene, and (2) a remote boreal forest site (Hyytiälä, Finland) during springtime which is predominantly influenced by monoterpene emissions. In both locations, the FIGAERO-HRToF-CIMS molecular composition observations explain at least $50 \%$ of the total AMS organic mass. That is, based on our declustering scans and distribution of binding enthalpies (e.g., Fig. 3c) from a similar chemical system (e.g., $\alpha$-pinene ozonolysis in the presence of $\mathrm{NO}_{x}$ ), we know that not all organic compounds are detected by iodide adduct ionization at the collision limit. It is reasonable to conclude that the underestimate compared to the AMS is because some compounds are detected less sensitively than we assume and not only because there are undetected components. Utilizing the declustering scans during a future field or chamber experiment would provide in situ constraints on how to better apply the collision-limited sensitivity to the mass spectra as illustrated with pinonic acid example above. Based on the results presented in Fig. 5, we conclude that the FIGAERO in combination with iodide adduct CIMS is able to detect the majority of oxidized organic molecules that thermally desorb from submicron organic aerosol, thereby providing novel and quantitatively

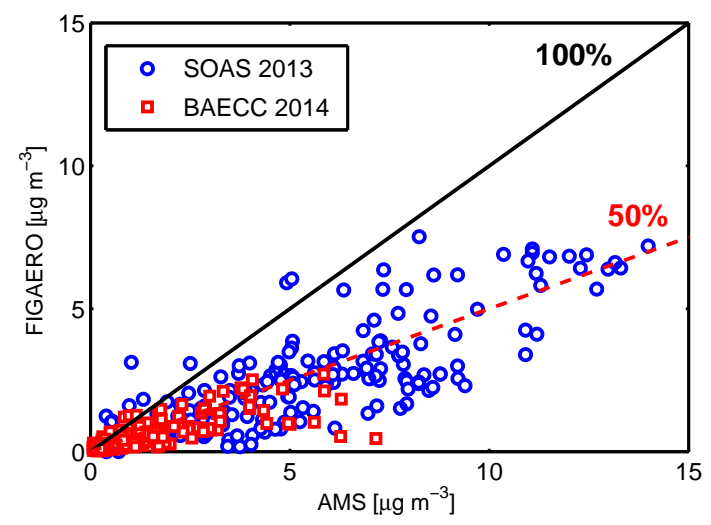

Figure 5. Application of the collision limit to secondary organic aerosol concentration utilizing a FIGAERO inlet. The application of the collision limit allows conversion of detected signals to a lower limit mass loading; despite this assumption, the mass loading explains a large fraction of SOA $(\sim 50 \%)$ and is highly correlated with total organic aerosol mass concentrations measured by an AMS $\left(R_{\mathrm{SOAS}}=0.82\right.$ and $\left.R_{\mathrm{BEACC}}=0.76\right)$. The lower correlation for BAECC is likely because of lower total organic and perhaps because the AMS was located on the ground, while the FIGAERO was located at the top of a $35 \mathrm{~m}$ tower. To attempt to reduce this potential bias, only data points during daytime are plotted for BAECC as surface inversions (below the tower) were regularly observed at night.

useful constraints on the molecular compositions responsible for SOA formation and growth.

\section{Conclusions}

We present a procedure that allows the determination of the collision-limited sensitivity of an iodide adduct chemical ionization mass spectrometer. We combine this limit with an experimental determination of the binding enthalpies of organic-iodide adducts to determine the extent to which collision-induced ion adduct dissociation (i.e., declustering) losses occur during transit through the ion optics of the instrument. We stress that the values of the collision limit or other calibration-derived sensitivity values reported herein are likely unique to the electric fields, IMR geometry, pressures and flows of our instrument. While useful as a relative guide, these values should not be applied to data from other instruments without conducting similar experiments as described here. In the case of adduct formation, steric factors and competitive ligand switching may affect the adduct formation rate, and therefore binding enthalpy alone does not determine sensitivity. For molecules containing a significant number of hydroxy (or hydroperoxy and carboxylic acid) groups, sterics probably play a minor role, so we expect the overall sensitivity to be collision limited and the adduct transmitted to the detector with minimal declustering losses. Many of these types of molecules are impossible 
to calibrate using traditional methods of authentic standards, but the combination of declustering scanning and collisionlimited sensitivity determination allows for reasonable constraints on the instrument response for hundreds of organic molecules which are now routinely detected in the atmosphere.

Acknowledgements. We would like to thank the Jimenez Group at the University of Colorado, Boulder for the use of AMS data from the SOAS campaign as well as the Virtanen Group at the University of Eastern Finland for AMS data from the BAECC campaign. We would also like to thank Tofwerk AG for useful discussions regarding the tuning of our mass spectrometer. We also thank the Cohen group for calibrating the output of our $\mathrm{N}_{2} \mathrm{O}_{5}$ calibration source using TD-LIF during the WINTER campaign. This work was supported by the US Department of Energy through awards from the Atmospheric System Research (DOE grant DE-SC0006867) and NSF (award number AGS-1360745).

Edited by: M. Sipilä

\section{References}

Aljawhary, D., Lee, A. K. Y., and Abbatt, J. P. D.: High-resolution chemical ionization mass spectrometry (ToF-CIMS): application to study SOA composition and processing, Atmos. Meas. Tech., 6, 3211-3224, doi:10.5194/amt-6-3211-2013, 2013.

Bertram, T. H., Thornton, J. A., and Riedel, T. P.: An experimental technique for the direct measurement of $\mathrm{N}_{2} \mathrm{O}_{5}$ reactivity on ambient particles, Atmos. Meas. Tech., 2, 231-242, doi:10.5194/amt-2-231-2009, 2009.

Bertram, T. H., Kimmel, J. R., Crisp, T. A., Ryder, O. S., Yatavelli, R. L. N., Thornton, J. A., Cubison, M. J., Gonin, M., and Worsnop, D. R.: A field-deployable, chemical ionization timeof-flight mass spectrometer, Atmos. Meas. Tech., 4, 1471-1479, doi:10.5194/amt-4-1471-2011, 2011.

Brophy, P. and Farmer, D. K.: A switchable reagent ion high resolution time-of-flight chemical ionization mass spectrometer for real-time measurement of gas phase oxidized species: characterization from the 2013 southern oxidant and aerosol study, Atmos. Meas. Tech., 8, 2945-2959, doi:10.5194/amt-8-2945-2015, 2015.

Brown, S. S., Stark, H., Ciciora, S. J., and Ravishankara, A. R.: In-situ measurement of atmospheric $\mathrm{NO}_{3}$ and $\mathrm{N}_{2} \mathrm{O}_{5}$ via cavity ring-down spectroscopy, Geophys. Res. Lett., 28, 3227-3230, doi:10.1029/2001GL013303, 2001.

Day, D. A., Wooldridge, P. J., Dillon, M. B., Thornton, J. A., and Cohen, R. C.: A thermal dissociation laser-induced fluorescence instrument for in situ detection of $\mathrm{NO}_{2}$, peroxy nitrates, alkyl nitrates, and $\mathrm{HNO}_{3}$, J. Geophys. Res., 107, ACH 4-1-ACH 4-14, doi:10.1029/2001JD000779, 2002.

DeCarlo, P. F., Kimmel, J. R., Trimborn, A., Northway, M. J., Jayne, J. T., Aiken, A. C., Gonin, M., Fuhrer, K., Horvath, T., Docherty, K. S., Worsnop, D. R., and Jimenez, J. L.: Field-Deployable, High-Resolution, Time-of-Flight Aerosol Mass Spectrometer, Anal. Chem., 78, 8281-8289, doi:10.1021/ac061249n, 2006.
Fortner, E. C., Zhao, J., and Zhang, R.: Development of Ion DriftChemical Ionization Mass Spectrometry, Anal. Chem., 76, 54365440, doi:10.1021/ac0493222, 2004.

Goldstein, A. H. and Galbally, I. E.: Known and unexplored organic constituents in the earth's atmosphere, Environ. Sci. Technol., 41, 1514-1521, doi:10.1021/es072476p, 2007.

Hearn, J. D. and Smith, G. D.: A Chemical Ionization Mass Spectrometry Method for the Online Analysis of Organic Aerosols, Anal. Chem., 76, 2820-2826, doi:10.1021/ac049948s, 2004.

Holzinger, R., Kasper-Giebl, A., Staudinger, M., Schauer, G., and Röckmann, T.: Analysis of the chemical composition of organic aerosol at the Mt. Sonnblick observatory using a novel high mass resolution thermal-desorption proton-transfer-reaction mass-spectrometer (hr-TD-PTR-MS), Atmos. Chem. Phys., 10, 10111-10128, doi:10.5194/acp-10-10111-2010, 2010.

Huey, L. G., Hanson, D. R., and Howard, C. J.: Reactions of SF6and I- with Atmospheric Trace Gases, J. Phys. Chem., 99, 50015008, doi:10.1021/j100014a021, 1995.

Iyer, S., Lopez-Hilfiker, F. D., Lee, B. H., Thornton, J. A., and Kurtén, T.: Modeling the detection of organic and inorganic compounds using iodide-based chemical ionization, The Journal of Physical Chemistry A, 120, 576-587, doi:10.1021/acs.jpca.5b09837, 2016.

Jokinen, T., Sipilä, M., Junninen, H., Ehn, M., Lönn, G., Hakala, J., Petäjä, T., Mauldin III, R. L., Kulmala, M., and Worsnop, D. R.: Atmospheric sulphuric acid and neutral cluster measurements using CI-APi-TOF, Atmos. Chem. Phys., 12, 4117-4125, doi:10.5194/acp-12-4117-2012, 2012.

Jordan, A., Haidacher, S., Hanel, G., Hartungen, E., Herbig, J., Märk, L., Schottkowsky, R., Seehauser, H., Sulzer, P., and Märk, T. D.: An online ultra-high sensitivity Proton-transfer-reaction mass-spectrometer combined with switchable reagent ion capability (PTR+SRI-MS), Int. J. Mass Spectrom., 286, 32-38, doi:10.1016/j.ijms.2009.06.006, 2009.

Junninen, H., Ehn, M., Petäjä, T., Luosujärvi, L., Kotiaho, T., Kostiainen, R., Rohner, U., Gonin, M., Fuhrer, K., Kulmala, M., and Worsnop, D. R.: A high-resolution mass spectrometer to measure atmospheric ion composition, Atmos. Meas. Tech., 3, 1039 1053, doi:10.5194/amt-3-1039-2010, 2010.

Kercher, J. P., Riedel, T. P., and Thornton, J. A.: Chlorine activation by $\mathrm{N}_{2} \mathrm{O}_{5}$ : simultaneous, in situ detection of $\mathrm{ClNO}_{2}$ and $\mathrm{N}_{2} \mathrm{O}_{5}$ by chemical ionization mass spectrometry, Atmos. Meas. Tech., 2, 193-204, doi:10.5194/amt-2-193-2009, 2009.

Lee, B. H., Lopez-Hilfiker, F. D., Mohr, C., Kurtén, T., Worsnop, D. R., and Thornton, J. A.: An Iodide-Adduct High-Resolution Time-of-Flight Chemical-Ionization Mass Spectrometer: Application to Atmospheric Inorganic and Organic Compounds, Environ. Sci. Technol., 48, 6309-6317, doi:10.1021/es500362a, 2014.

Lopez-Hilfiker, F. D., Mohr, C., Ehn, M., Rubach, F., Kleist, E., Wildt, J., Mentel, Th. F., Lutz, A., Hallquist, M., Worsnop, D., and Thornton, J. A.: A novel method for online analysis of gas and particle composition: description and evaluation of a Filter Inlet for Gases and AEROsols (FIGAERO), Atmos. Meas. Tech., 7, 983-1001, doi:10.5194/amt-7-983-2014, 2014.

Lopez-Hilfiker, F. D., Mohr, C., Ehn, M., Rubach, F., Kleist, E., Wildt, J., Mentel, Th. F., Carrasquillo, A. J., Daumit, K. E., Hunter, J. F., Kroll, J. H., Worsnop, D. R., and Thornton, J. A.: Phase partitioning and volatility of secondary or- 
ganic aerosol components formed from $\alpha$-pinene ozonolysis and $\mathrm{OH}$ oxidation: the importance of accretion products and other low volatility compounds, Atmos. Chem. Phys., 15, 7765-7776, doi:10.5194/acp-15-7765-2015, 2015.

Slusher, D. L.: A thermal dissociation-chemical ionization mass spectrometry (TD-CIMS) technique for the simultaneous measurement of peroxyacyl nitrates and dinitrogen pentoxide, J. Geophys. Res., 109, D19315, doi:10.1029/2004JD004670, 2004.

Veres, P., Roberts, J. M., Warneke, C., Welsh-Bon, D., Zahniser, M., Herndon, S., Fall, R., and de Gouw, J.: Development of negative-ion proton-transfer chemical-ionization mass spectrometry (NI-PT-CIMS) for the measurement of gas-phase organic acids in the atmosphere, Int. J. Mass Spectrom., 274, 48-55, doi:10.1016/j.ijms.2008.04.032, 2008.

Veres, P., Roberts, J. M., Burling, I. R., Warneke, C., de Gouw, J., and Yokelson, R. J.: Measurements of gas-phase inorganic and organic acids from biomass fires by negative-ion proton-transfer chemical-ionization mass spectrometry, J. Geophys. Res., 115, D23302, doi:10.1029/2010JD014033, 2010.
Yatavelli, R. L. N., Lopez-Hilfiker, F., Wargo, J. D., Kimmel, J. R., Cubison, M. J., Bertram, T. H., Jimenez, J. L., Gonin, M., Worsnop, D. R., and Thornton, J. A.: A Chemical Ionization High-Resolution Time-of-Flight Mass Spectrometer Coupled to a Micro Orifice Volatilization Impactor (MOVI-HRToF-CIMS) for Analysis of Gas and Particle-Phase Organic Species, Aerosol Sci. Tech., 46, 1313-1327, doi:10.1080/02786826.2012.712236, 2012.

You, Y., Kanawade, V. P., de Gouw, J. A., Guenther, A. B., Madronich, S., Sierra-Hernández, M. R., Lawler, M., Smith, J. N., Takahama, S., Ruggeri, G., Koss, A., Olson, K., Baumann, K., Weber, R. J., Nenes, A., Guo, H., Edgerton, E. S., Porcelli, L., Brune, W. H., Goldstein, A. H., and Lee, S.-H.: Atmospheric amines and ammonia measured with a chemical ionization mass spectrometer (CIMS), Atmos. Chem. Phys., 14, 12181-12194, doi:10.5194/acp-14-12181-2014, 2014.

$\mathrm{Yu}, \mathrm{H}$. and Lee, S.-H.: Chemical ionisation mass spectrometry for the measurement of atmospheric amines, Environ. Chem., 9, 190, doi:10.1071/EN12020, 2012. 